\title{
CASA ASF + PROTOTIPO DE VIVIENDA AUTOSUFICIENTE PARA SANTA FE
}

Bertuzzi Alfredo, Boaglio Sebastián, Gutierrez Jesús

Taller de Tesis, Facultad de Arquitectura, Diseño y Urbanismo, Universidad Nacional del Litoral

Santa Fe, Argentina

Palabras clave: Arquitectura, sustentabilidad, energía, renovables, autosuficiencia.

\section{Resumen:}

Los sistemas de infraestructura actuales a los que tenemos conectadas nuestras viviendas producen un daño irreversible a nuestro planeta. La producción de energía eléctrica crea un impacto negativo en el medio ambiente generando contaminación atmosférica, producción de $\mathrm{CO} 2$, entre otros. Para minimizar este impacto ambiental negativo, se propone como objetivo general conocer y aplicar estrategias de diseño bioclimático y de eficiencia energética para el diseño de un prototipo de vivienda autosuficiente, capaz de abastecerse de recursos y energías renovables, para la ciudad de Santa Fe. Para lograr dicho objetivo, se indagan pautas de arquitectura bioclimática aplicables en la Zona Bioambiental IIB e investigan sistemas que funcionen mediante energías renovables, como también estrategias de reducción de consumo, reutilización y reciclaje de residuos y recursos.

Como resultado, esta vivienda fue diseñada con tecnologías y estrategias de arquitectura bioclimática aplicada a un clima Cálido - Húmedo. Sustentable en su materialidad, construcción, uso y manejo de desechos y recursos, y totalmente autosuficiente en agua y energía, la vivienda se independiza del sistema infraestructural y redes urbanas, logrando cero emisiones de CO2. La misma genera su energía mediante fuentes renovables, produciendo más energía de la demanda requerida, clasificándose así como vivienda de "energía plus". El sobrante se inyecta a la red entregando energía limpia al sistema, reduciendo la producción eléctrica proveniente de combustibles fósiles, y con ello la contaminación ambiental.

Como verificación y conclusión se realiza un etiquetado edilicio comparándola con una vivienda tradicional en varios aspectos: Energía, Materialidad, Consumo de agua y presupuesto.

\section{Introducción al tema}

El tema de la arquitectura autosuficiente, bioclimática y sustentable, es una faceta de esta disciplina sumamente relevante en la actualidad y más aún en el futuro veni $\neg$ dero. En las últimas décadas nuestro planeta ha venido sufriendo numerosas trans $\neg$ formaciones socio-naturales que han contribuido con el deterioro del medio ambien $\neg$ te que actualmente padecemos.

Varios autores definen estos conceptos, que a su vez son muy similares. Tomamos el que nos pareció más adecuado para aclarar su significado. Czajkowski y Gómez definen la arquitectura sustentable como "un modo de concebir la arquitectura bus $\urcorner$ cando aprovechar los recursos naturales de tal modo que se minimice el impacto ambiental de las construcciones sobre el ambiente natural y sobre los habitantes" y la diferencian de la arquitectura bioclimática como el "procedimiento y método por el cual se puede alcanzar una Arquitectura Sustentable".

Para que una arquitectura sea considerada sustentable, se debe tener en cuenta: las condiciones climáticas, la hidrografía y los ecosistemas del entorno, la eficacia y moderación en el uso de materiales de construcción, la reducción del consumo de energía para calefacción, refrigeración, iluminación, utilización de fuentes de energía renovables, confort higrotérmico, salubridad y habitabilidad de las edificaciones.

Según Víctor Olgyay "la arquitectura bioclimática es un tipo de arquitectura donde el equilibrio 
y la armonía son una constante con el medio ambiente. Se busca lograr un gran nivel de confort térmico, teniendo en cuenta el clima y las condiciones del entorno para ayudar a conseguir el confort térmico interior mediante la adecuación del diseño, la geometría, la orientación y la construcción del edificio adaptado."

El uso y el consumo de recursos y energías no renovables en las viviendas, al estar conectadas al sistema de infraestructuras de la ciudad, favorecen a un impacto nega 7 tivo al ambiente. Como sostiene Michael Reynolds: "también estamos muriendo de esta manera. Los sistemas nos dan energía por un lado y veneno por el otro. Lluvia ácida, desperdicios radioactivos, telarañas de transmisión eléctrica, ríos y mares con ᄀtaminados, vida salvaje desvaneciéndose, todo esto es parte del precio de los sopor $\neg$ tes de vida necesarios para que el concepto de vivienda actual siga siendo funcional."

Aquí radicó nuestra preocupación y motivación que, a través de la arquitectura, se pueda contribuir a disminuir éste impacto que se produce con la generación de de $\neg$ sechos y el consumo de energías y recursos no renovables, y así, no seguir agravando el cambio climático.

\section{Situación problema}

Las viviendas necesitan estar conectadas a infraestructuras para poder funcionar y ser habitables. Son totalmente dependientes de los sistemas que nos abastecen de recursos y energías (gas, agua, cloacas y energía eléctrica). Los recursos y sus tra ᄀtamientos dependen de los sistemas eléctricos para funcionar y no se contempla su reutilización y/o reciclaje. Las energías que se proveen mediante la infraestructura actual provienen de fuentes no renovables además que, para ser generadas, conta $\neg$ minan el medio ambiente.

A su vez, la ausencia de un diseño bioclimático en una vivienda puede lograr que ésta consuma cinco veces más la cantidad de energía necesaria para lograr el confort pretendido. Desaprovechar las características del entorno al momento de construir, sin pensar en la orientación y no incorporar materiales aislantes adecuados, aumenta en forma sustancial el consumo de energía.

Por estos factores, la dependencia de las viviendas actuales a los sistemas infraes $\neg$ tructurales de la ciudad contribuye a un impacto ambiental negativo siendo uno de los elementos que favorecen al cambio climático global.

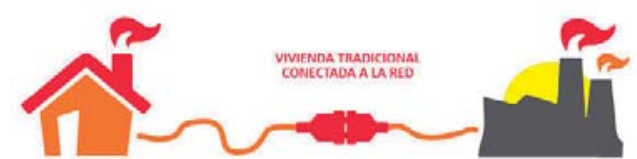

Figura 1.

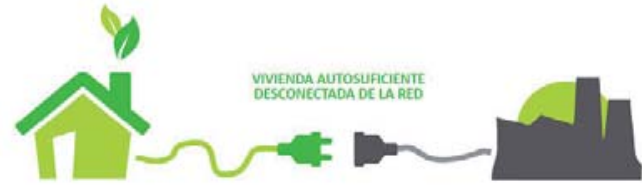

Figura 2.

Vivienda Tradicional conectada a la red Vivienda Autosuficiente desconectada de la red

\section{Estado del arte}

\subsection{Cambio Climático}

El hombre ha producido de una manera $u$ otra, un deterioro en el ambiente desde el inicio de sus tiempos. Cuando el número de habitantes en el planeta era bajo, el impacto que producía también lo era y el ecosistema era capaz de digerir estas accio $\neg$ nes haciéndolas imperceptibles para el planeta. A medida que la raza humana se fue expandiendo comenzó a dañar el planeta indiscriminadamente con el desarrollo de su existencia de manera inintencionada.

Hoy, con el planeta colmado de humanos, realizando sus actividades sociales, co $\neg$ merciales, industriales, etc., el medioambiente ya no es capaz de asimilar los daños que se le producen. El impacto ambiental ya es enorme y ha desequilibrado el ecosis $\neg$ tema global y los diferentes ecosistemas locales. 
Como consecuencia de esto, el deterioro medioambiental ya es perceptible de forma directa para el ciudadano medio, con una variada gama de complicaciones e inestabilidades medioambientales que se han presentado como el calentamiento global, la contaminación atmosférica, la contaminación de acuíferos, la escasez de agua, la salinización de los océanos, la lluvia ácida, la contaminación de muchas $\cos \urcorner$ tas, los vertidos marinos, los vertidos en acuíferos, los vertidos terrestres, el desorden climático, la desaparición de especies, la escasez de zonas verdes, el aumento de la desertificación, la falta de impermeabilización de suelos, la escasez de recursos, el aumento de residuos, etc.

\subsection{Consecuencias globales del cambio climático}

El sol transmite calor mediante la radiación solar, propiamente dicha. Gran parte de este calor se absorbe a nivel de la superficie para luego convertirse en calor terres $\neg$ tre. A su vez, la tierra tiene la capacidad de irradiar calor hacia el exterior.

Vapor de agua, dióxido de carbono, metano, óxido nitroso y ozono son algunos de los gases que componen la atmosfera; la concentración de estos gases los hace hábiles para absorber el calor de la radiación de la Tierra. La existencia de estos Gases de Efecto Invernadero en la troposfera, la parte baja de la atmosfera, favorece a que el calor que la superficie terrestre transmite sea contenido y resulte en la elevación de las temperaturas terrestres. Este proceso es conocido como Efecto Natural de In $\urcorner$ vernadero. Sin este mecanismo la temperatura media de la Tierra seria considerable $\neg$ mente más baja haciéndola inhabitable para los seres humanos.

Por su parte, el Sistema Climático, es el conjunto e interacción de diferentes ele $\neg$ mentos propios de nuestro planeta como las superficies cubiertas por tierra, hielo y agua, la vegetación, los animales y todos los otros seres vivos y la propia atmósfera, elementos que interactúan y relacionan entre si de manera constante. El Clima es la manifestación del equilibrio existente dentro de este Sistema Climático.

El calentamiento global es inminente y desde la década del ' 50 han surgido nue $\neg$ vos episodios sin precedentes en los últimos mil años. El aire y el agua se han calen $\neg$ tado, los volúmenes de hielo y nieve han disminuido y por ello el nivel del mar ha aumentado y las concentraciones de gases de efecto invernadero han crecido signi $\neg$ ficativamente.

La superficie terrestre en las tres últimas décadas ha sido continuamente más cáli $\neg$ da que las décadas anteriores. Los mantos de hielo de la Antártida y Groenlandia han perdido masa de manera considerable en las últimas décadas y los glaciares se han retraído continuamente en casi todo el planeta.

El nivel del mar se ha elevado a un ritmo acelerado ya desde mediados del 1800, siendo superior a la media de los milenios anteriores, es así que durante el período 1901-2010, el nivel medio global del mar se elevó 0,19m.

Las concentraciones atmosféricas de gases efecto invernadero como dióxido de carbono, metano y óxido nitroso han aumentado sus niveles en los últimos siglos. Debido a las emisiones emanadas de los combustibles fósiles y la vorágine del desa $\neg$ rrollo industrial, el dióxido de carbono aumentó desde la era preindustrial, no sólo contaminando el aire sino también contribuyendo a la acidificación de los océanos por la absorción de dichos gases.

Figura 3. Efecto Invernadero

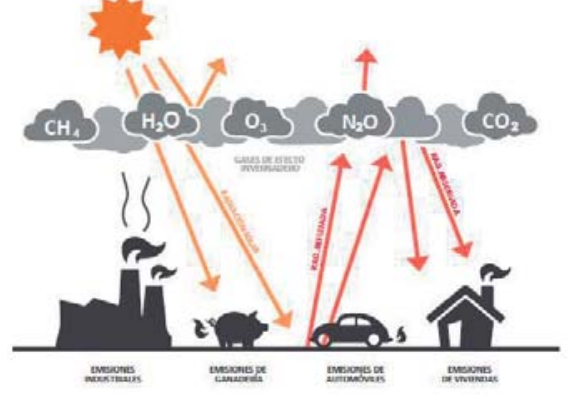




\section{$4 \quad$ Metodología de trabajo}

Como pregunta de investigación nos cuestionamos: ¿̇s posible minimizar el impacto ambiental negativo que produce la dependencia de las viviendas actuales a los sistemas infraestructurales de Santa $\mathrm{Fe}$, y conseguir un ahorro económico a largo plazo, mediante el desarrollo de un prototipo autosuficiente?

Como objetivo principal nos propusimos proyectar un prototipo de vivienda autosuficiente para el ámbito local, en base a una investigación de las distintas tecnologías y estrategias aplicables de la arquitectura bioclimática, sustentable y de eficiencia energética y de agua. Este prototipo debería contemplar lo necesario para desarrollar la autonomía en una vivienda, es decir, que se pueda abastecer por sí misma, de forma independiente a la conexión de las infraestructuras y redes, a través del aprovechamiento de las energías renovables, estrategias de aho $\neg$ rro de agua y energía y empleando además los conceptos de sustentabilidad y diseño bioclimático.

Para lograr dicho objetivo tuvimos que indagar las pautas de arquitectura bioclimática que se puedan aplicar en la Zona Bioambiental que abarque a la Ciudad de Santa Fe y su zona metropolitana; investigar los diferentes sistemas que funcionen mediante energías renovables que se puedan aplicar al área de estudio: energía solar, eólica, climatización geotér $\neg$ mica y la optimización de recursos; indagar estrategias de reducción de consumo, reutilización y reciclaje de residuos y recursos; y explorar las Normativas vigentes y regulaciones en el marco jurídico de la Arquiᄀtectura Bioclimática y Sustentable; y definir los sistemas de energías renovables, dispositivos inteligentes y optimiza $\neg$ ción de recursos más adecuados para la zona a intervenir.

\section{Arquitectura autosuficiente}

Actualmente, la arquitectura utiliza los recursos de la naturaleza y los devuelve en forma de desecho, producto de su actividad, considerándolo como un proceso lineal y finito.

Esto produce enormemente una degradación en el ecosistema. Como contraparte, la naturaleza funciona de una manera cíclica e infinita, obtiene sus propios recursos que necesita (energía y agua), los utiliza y los regenera para un nuevo uso. Este concepto es la clave principal para una Arquitectura Autosuficiente. Una arquitectura de estas características debe lograr producir, de la misma manera que la naturaleza, su propia energía y agua de una manera sustentable, sin necesitar estar conectadas a las redes de suministros de la ciudad.

Para lograr una autosuficiencia en ambos casos (energía y agua) es necesario que el edificio consuma la menor cantidad y al menor costo posible, generando así lo mínimo necesario.

\section{Resultados obtenidos}

La Casa ASF+ es un prototipo de vivienda autosuficiente en energía y agua, la cual se desarrolla a base de estrategias de diseño bioclimático, de ahorro energético y de agua, con la implementación de energías renovables para su total auto-abastecimiento, independiente de los sistemas infraestructurales de la ciudad y la región. Esta vivienda, además, produce más energía de la que consume, convirtiéndola en una vivienda de energía plus, posibilitando inyectar esta energía limpia a la red, y así también obtener un beneficio económico por ello. Al estar desconectados, se reduce el impacto ambiental negativo que se genera para la producción y traslado de los servicios de energía eléctrica, agua, gas y cloaca, disminuyendo el consumo de combustibles fósiles, y con ello la contaminación ambiental. 


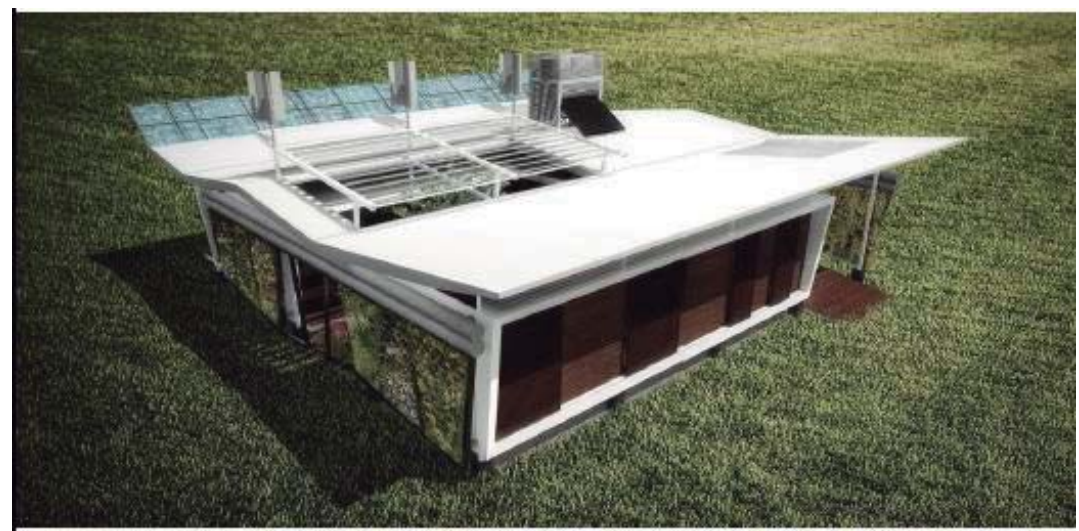

Figura 4. Perspectiva a vuelo de pájaro CASA ASF+.

Sustentable en su materialidad, construcción, uso y manejo de desechos y recursos, y totalmente autosuficiente en energía y agua, de forma independiente a la conexión de las infraestructuras y redes urbanas, con cero emisiones de $\mathrm{CO} 2$, ya que produce energía del aprovechamiento de fuentes renovables (sol, viento, tierra). La vivienda está diseñada en relación a un clima Cálido Húmedo, con soluciones constructivas y estrategias de diseño para tal situación.

Prototipo para ser ocupado por familias de 3 a 6 personas. Familias jóvenes, con una forma de coexistir con la naturaleza que hoy ya es una tendencia mundial, y estarán interesadas en contribuir con el medio ambiente.

La Casa ASF+ tiene grandes beneficios ambientales, sociales, energéticos y económicos. A medida que se multiplique en el territorio:

- Mayor será la relación con el medio ambiente, ya que la vegetación es un aspecto muy relevante en su diseño, transformándose en núcleos naturales generadores de $\mathrm{O} 2$.

- Mayor será la reducción de emisiones de CO2, ya que ésta vivienda no produce emisiones, con un elevado ahorro energético y de agua, utilizando fuentes renovables de energía.

- Se preverá el riesgo de inundaciones a causa de lluvias torrenciales, actuando las mismas como retardadores y recolectores pluviales.

- Mayor será la entrega de energía limpia a la red eléctrica de la ciudad, reduciendo la contaminación que se genera para la producción de la misma.

- Mayor será la reducción de la contaminación que genera la producción y traslado de los servicios (energía, gas, agua, cloaca), ya que no se los utiliza.

- Mayor será el beneficio medioambiental al utilizar materiales no tóxicos, re-utilizables, reciclables y con bajo contenido energético.

- Los cortes de luz por falta de capacidad eléctrica, se minimizarán, ya que las Casas ASF+ tienen una máxima producción de energía eléctrica en verano, momento crítico del año donde tienen lugar los mismos, funcionando como núcleos productores de energía, descongestionando así la central eléctrica; y en el caso de ocurrir el corte propiamente dicho, estas viviendas no serán afectadas.

- Mayor será la calidad de vida, con un óptimo confort higrotérmico, salubridad, y habitabilidad de las edificaciones, en un espacio en contacto con la naturaleza, con materiales verdes, y utilizando energías renovables. Las personas pasan el $85 \%$ de su tiempo en las viviendas, por lo que la calidad de este ambiente es esencial. La mejora del aislamiento térmico de las viviendas va acompañado a la vez, por aislamiento sonoro.

- Estas viviendas ayudan a proteger la biodiversidad al cuidar los espacios abiertos, restaurando sitios ecológicamente dañados y promoviendo el cuidado ambiental.

- Se obtendrá un ahorro económico a largo plazo, amortizándose en un plazo entre cinco y 
ocho años, después del cual, sólo producirá ganancias.

\section{Etiquetado edilicio y comparación}

Se realizó una comparación de la etiquetación edilicia de los siguientes casos en relación a tres factores (gasto energético, consumo de agua y presupuesto):

Casa ASF+: Vivienda autosuficiente de energía plus (caso de aplicativo)

Casa EE: Vivienda tradicional totalmente eléctrica (caso comparativo)

Para el gasto energético total de cada vivienda, tomaremos como base para la comparación de las mismas, el índice de prestación energética (IPE), expresado en $\mathrm{Kwh} / \mathrm{m} 2 /$ año, que engloba el consumo correspondiente a re $\neg$ frigeración en verano, calefacción en invierno, iluminación y agua caliente sanitaria, y las emisiones de $\mathrm{CO} 2$ que la vivienda produce para satisfacer estas demandas en un año. Estos valores son fijos para cada vivienda. A su vez, determinaremos el consumo total de una vivienda en un año, sumado este valor al gasto correspondiente a los electrodomésticos utilizados y los hábitos de consumo diario de una familia tipo de cuatro personas. Estos valores son relativos, variables. Se tendrán en cuenta artefactos de eficien $\neg$ cia $A$ y hábitos de consumo conscientes, enfocados en el ahorro energético para la Casa ASF+, diferenciándola a la de una vivienda tradicional, donde los electrodomésticos y los hábitos de consumo son ineficientes.

El consumo de agua anual correspondiente a una vivienda tradicional es mayor, en relación al de la Casa ASF+, ya que esta última gestiona de mane $\neg$ ra eficiente este recurso: recolecta aguas de lluvia, reutiliza aguas grises y tiene artefactos que reducen en gran medida el consumo de agua potable.

Por último, se comparará el presupuesto final de ambas viviendas, te $\neg$ niendo en cuenta la materialidad, equipamiento (sistemas de refrigeración, calefacción, agua caliente sanitaria), instalaciones, y trabajos complemen ᄀtarios para desarrollarlas. En el caso de la Casa ASF+ se cuenta el beneficio anual, proveniente de la venta de energía limpia a la red, producida por energías renovables. La misma tendrá un presupuesto mayor que la tradi $\neg$ cional, pero se amortizará a corto plazo, por los altos valores de servicios, por el beneficio producido, por la ineficiencia energética en la climatización y utilización de electrodomésticos y en el uso desmedido del agua, que $\mathrm{ca}$ ᄀracterizan a una vivienda tradicional.

\subsection{Comparación de viviendas}

Como primera medida, la Casa ASF+ se diseñó de acuerdo a las condiciones climáticas de Santa $\mathrm{Fe}$, sus vientos predominantes, ángulos solares en las distintas estaciones, temperaturas, etc. A con ᄀtinuación se estudió la materialidad, para llegar a un nivel de eficiencia energética $A, y$ de esta ma $\neg$ nera reducir en gran medida el consumo total de la vivienda correspondiente a climatización, ilumi $\neg$ nación y agua caliente sanitaria (IPE), el cual que se proporcionó por medio de energías renovables.

De esta manera, se analizan los distintos materiales de la envolvente y se determina el coeficien ᄀte de transmitancia térmica $\mathrm{K}$ y la variación media ponderada de temperatura entre el interior y el exterior $(\mathrm{Tm})$, lo que define el nivel de eficiencia energética de la envolvente. La Casa ASF+ tiene un $\mathrm{K}$ de $0,30 \mathrm{w} / \mathrm{m} 2 . \mathrm{k}$ con un nivel de eficiencia energética de la envolvente $\mathrm{A}$, ya que su $\mathrm{Tm}=0,77{ }^{\circ} \mathrm{C}$, menor a 1. La Casa EE posee un $\mathrm{K}$ de $2,28 \mathrm{w} / \mathrm{m} 2 . \mathrm{k}$ con un nivel de eficiencia energética de la envolvente $H$, siendo su $\mathrm{Tm}=4,69^{\circ} \mathrm{C}$.

El siguiente paso es comparar el Índice de Prestación Energética (IPE). En relación al consumo energético, en lo que refiere a climatización, iluminación y agua caliente sanitaria, la Casa EE tiene un IPE de $87,2 \mathrm{Kwh} / \mathrm{m} 2 /$ año y la Casa ASF+ 3,72 Kwh/m2/año. Si tenemos en cuenta el consumo eléctrico total, contan $\urcorner$ do la totalidad de los electrodomésticos, la Casa EE tiene un consumo eléc- 
trico de 125,83 Kwh/m2/ año y la Casa ASF+ un consumo total de 16,37 Kwh/m2/año.

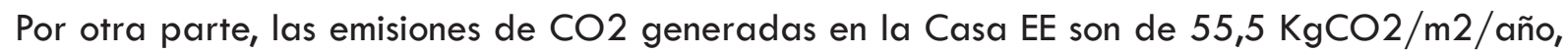
cuando en la Casa ASF+ es cero. Es decir, que se reduce hasta un $95 \%$ el consumo eléctrico y $100 \%$ las emisiones de CO2.

En cuanto al consumo de agua potable total de la Casa EE, se consume 596.775 litros por año, mientras que la Casa ASF+ consume 273.750 litros. Con un uso consciente del agua y con la utilización de dispositivos eficientes de reducción del consumo, se reduce en un $55 \%$ el uso del agua total, en relación a una vivienda tradicional. Sumadas estrategias de reutilización de aguas grises y de aguas de lluvia, se llega a reducir el consumo total, utilizando solo 175.200 litros de agua potable, disminuyendo hasta un $70 \%$ el uso de agua potable de nuestro prototipo en relación a las demás viviendas.

Por último, con respecto al presupuesto, la Casa ASF+ es un $5 \%$ más costosa en relación a una vivienda tradicional, sin computar los sistemas de generación de energía renovable, climatización y agua caliente sanitaria de ambas opciones. Si tenemos en cuenta estos sistemas que hacen operativas a las viviendas, el valor total de la Casa ASF+ es un $28 \%$ mayor al de una vivienda tradicional. La misma se amortizará en un plazo de 5 a 8 años, sin tener en cuenta el mayor valor de mercado para compraventa y alquiler del inmueble que poseerá al estar autoabastecida por energías renovables y ser sustentable.
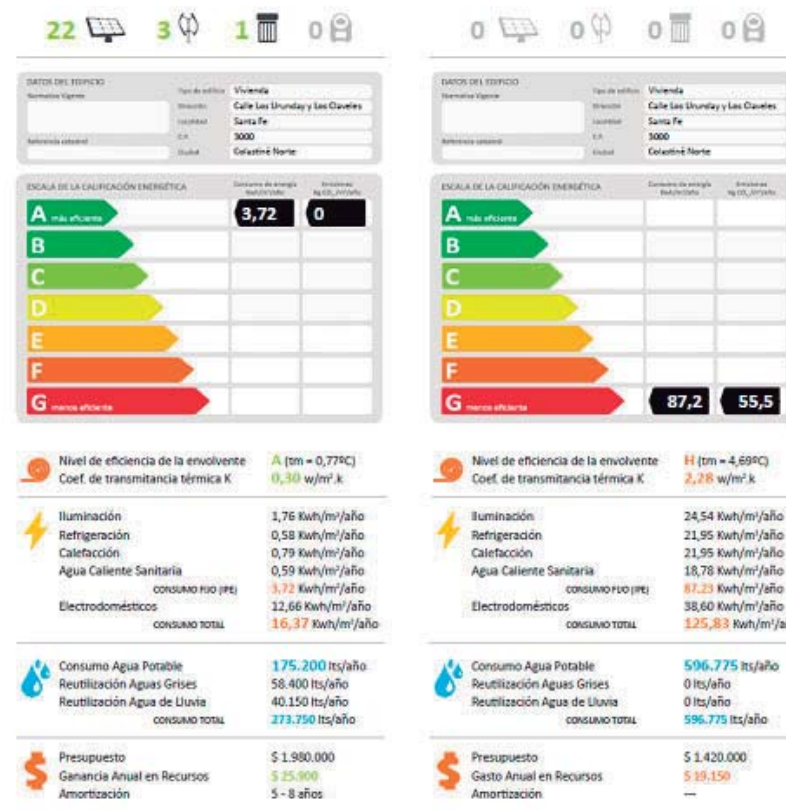

Figura 5. Etiquetado edilicio CASA ASF+

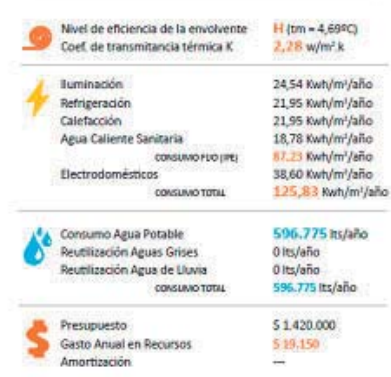

Figura 6. Etiquetado edilicio casa tradicional eléctrica.

\section{Conclusión}

Para minimizar el impacto ambiental negativo que se produce con la generación de desechos y el consumo de energías y recursos no renovables, mediante la depen $\neg$ dencia de las viviendas actuales a los sistemas infraestructurales de Santa $\mathrm{Fe}$, se desa $\neg$ rrolló el prototipo de vivienda autosuficiente de energía plus Casa ASF+. Esta vivienda está diseñada con tecnologías y estrategias de arquitectura bioclimática aplicada al clima de la ciudad de Santa Fe. Sustentable en su materialidad, construcción, uso y manejo de desechos y recursos, y totalmente autosuficiente en energía y agua, de forma independiente a la conexión de las infraestructuras y redes urbanas, con emi $\neg$ siones cero de $\mathrm{CO} 2$. Esta vivienda produce energía del aprovechamiento de fuentes renovables (sol, viento, tierra), generando más energía que la demanda requerida, clasificándose así como vivienda de "energía plus". Dicha energía se utilizará para in ᄀyectarla a la red eléctrica, y de

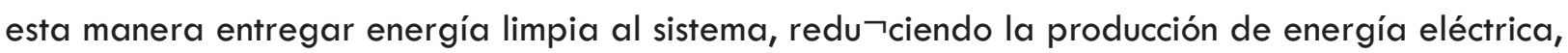
disminuyendo el consumo de combustibles fósiles, y con ello la contaminación ambiental. 


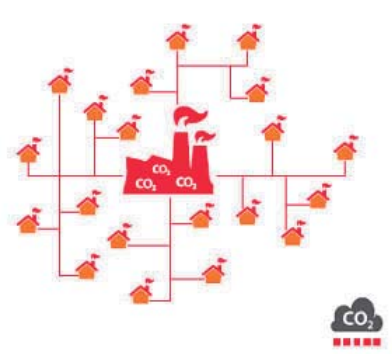

Figura 7.

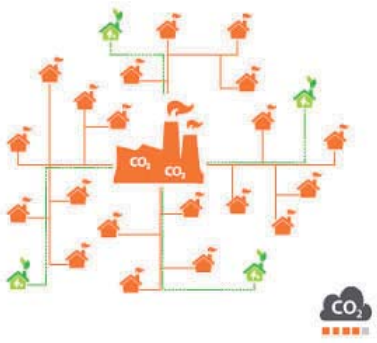

Figura 8.

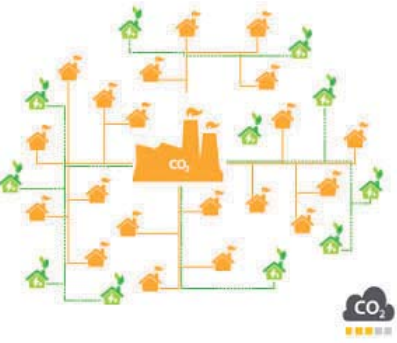

Figura 9.

Situación actual Inserción media de Casas ASF+ Inserción alta de Casas ASF+

En base al trabajo de investigación realizado, se comprueba que es posible y totalmente factible minimizar sustancialmente el impacto ambiental negativo que produce la de $\neg$ pendencia de las viviendas actuales a los sistemas infraestructurales de Santa Fe, y así conseguir un ahorro económico a largo plazo, mediante el desarrollo de un prototipo autosuficiente, desconectado de estos sistemas: La casa ASF+.

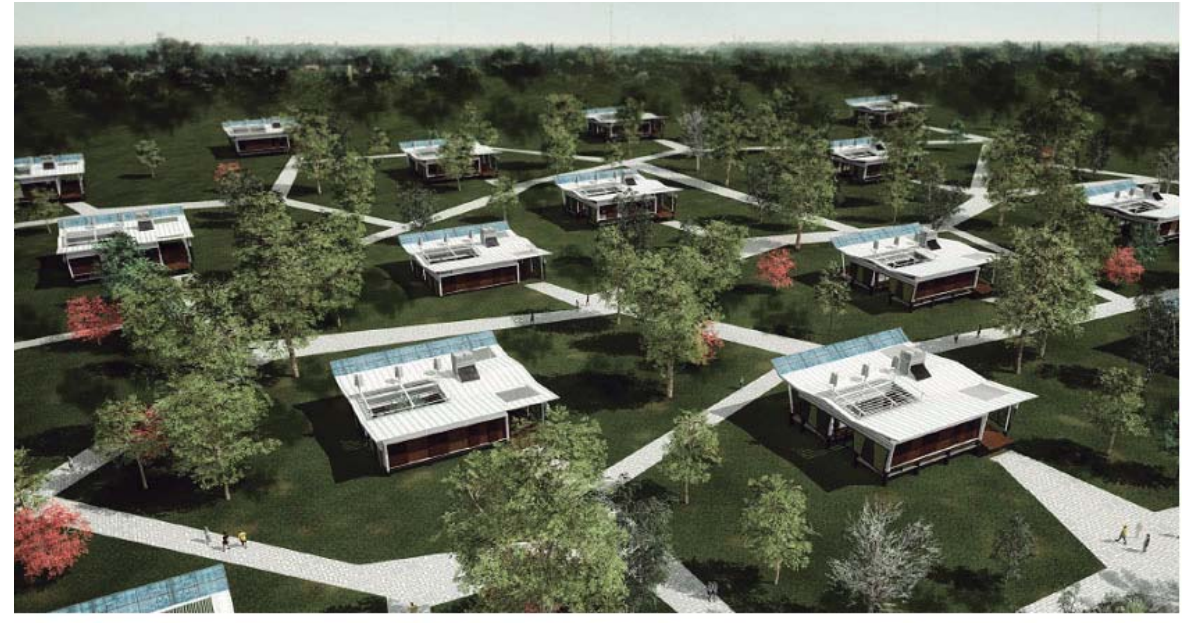

Figura 10. Casas ASF+ multiplicadas en el territorio

\section{Bibliografía}

REYNOLDS, Michael. "Nave Tierra Volumen I" Edición Hemisferio Sur, 2011

CZAJKOWSKI, Jorge, GÓMEZ Analía. "Arquitectura Sustentable". Editorial Arte Gráfico Editorial Argentino. Buenos Aires, 2009.

OLGYAY, Víctor. "Arquitectura y clima: Manual de diseño bioclimático para arquitectos y urbanistas". Gustavo Gili. 1998.

EVANS, Martín. "Sustentabilidad en Arquitectura I". Editorial CPAU. Buenos Aires, 2010.

DE GARRIDO, Luis. "Un nuevo paradigma en Arquitectura: Luis de Garrido, naturalezas artificiales 2001-2012". Instituto Monsa de Ediciones. 2012.

CARLI, César Luís. " $8^{\circ}$ al Sur del Trópico de Capricornio". Sainte Claire Editora S.R.L. Buenos Aires. 1980. 